erscheinen, daß große Geschwindigkeiten in der ersten Drift zahlreicher auftreten, denn Turner verlegt das Zentrum des Systems in den Vertex und Drift II enthält also die auf dem Rückweg befindlichen Sterne, welche das Zentralgebiet durchmessen haben, Drift I aber die von außen kommenden.

Es ist zu hoffen, daß schon in naher Zukunft ausreichendes Material an Totalbewegungen für eine direkte Prüfung der dynamischen Verhältnisse des Sternsystems zu erlangen ist. Für 278 Sterne meines I9 I 3 abgeschlossenen
Parallaxenkataloges liegen, schon jetzt brauchbare Radialgeschwindigkeiten vor, und eine strenge Berechnung der Totalbewegung für die Argumente $\pi-\mathrm{d} \pi, \pi$ und $\pi+\mathrm{d} \pi$ wird gestatten, die brauchbaren Elemente von den illusorischen Werten abzutrennen. Ein besonders rascher Fortschritt wäre zu erzielen, wenn die Beobachter von Parallaxen nur Sterne mit bereits gemessener Radialgeschwindigkeit auf ihr Programm setzen würden.

Wien, I 9 I 4 Juli 24.

Robert Klumak.

\title{
Osservazioni di comete
}

al equatoriale Steinheil-Cavignato del R. Oss. Coll. Romano.

\begin{tabular}{|c|c|c|c|c|c|c|c|c|c|c|c|c|}
\hline I 9 I & & $\begin{array}{c}\text { T. m. } \\
\text { Roma C. R. }\end{array}$ & $1 \alpha$ & $.1 \delta$ & Cf. & Oss. & $\alpha$ app. & $\log p \cdot \Delta$ & $\delta$ app. & $\log p \cdot \Delta$ & Red. ad 1. app. & $*$ \\
\hline \multicolumn{13}{|c|}{ Cometa rgr 3 f (Delavan). } \\
\hline ugl. & & $\mathrm{I} 4^{\mathrm{h}} 33^{\mathrm{m}} 55^{\mathrm{s}}$ & $+4^{\mathrm{m}} 49^{\mathrm{s}} \cdot 2 \mathrm{I}$ & $+I^{\prime} 54.0$ & 10.2 & M & $5^{\mathrm{h}} 42^{\mathrm{m}} 33^{\mathrm{s}} \cdot 99$ & $9.720_{n}$ & $+36^{\circ} 23^{\prime}$ I $6^{\prime \prime} \mathrm{I}$ & 0.757 & $+\mathrm{I} .48+6.2$ & I \\
\hline ett. & I 5 & 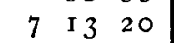 & - I $\quad$ I 7.63 & +5 I $8.5:$ & $\mathrm{I} 8.2$ & M & Io $\circ 6.39$ & $9.7 \circ \mathrm{I}$ & $+495 \mathrm{I}$ I.7: & 0.789 & $+2.08-10.5$ & 2 \\
\hline & 20 & $6424 I$ & - I 25.34 & $+\mathrm{I} 56.2$ & 10.2 & M & I0 $3^{8} \quad 23.3^{8}$ & $9.7^{6 \mathrm{I}}$ & $\begin{array}{lll}+49 & 1 & 33.3\end{array}$ & $0.80 \mathrm{I}$ & $+1.92-12.0$ & \\
\hline & 27 & $\begin{array}{lll}6 & 4 \times & 46\end{array}$ & -129.65 & -. 029.4 & 20.2 & M & I I 3 I 9 & 57 & $+463^{6} 27.1$ & 71 & $+1.67-$ & 4 \\
\hline Ott. & 2 & $6363 I$ & $-0 \quad 53$ & to 37.0 & 20.3 & M & I $26 \quad$ I 7 & $6 \mathrm{I}$ & +44244.1 & $5 \mathrm{I}$ & $53-14.1$ & \\
\hline & 7 & $\begin{array}{lll}6 & 22 & 42\end{array}$ & +o 55.74 & $-7 \quad 14.4$ & I 6.3 & M & 12 $38 \quad$ I 6.99 & 9.754 & $+40553^{6.2}$ & 0.723 & $+1.44-14.8$ & 6 \\
\hline & I 3 & $\begin{array}{lll}6 & 27 & 48\end{array}$ & +o $10.3 \mathrm{I}$ & $+\mathrm{I} 5 \mathrm{I} .0$ & 20.2 & $\mathrm{M}$ & - & 9.729 & - & $0.73^{2}$ & $+1.3^{6}-14.6$ & 7 \\
\hline Nov. & 10 & $\begin{array}{lll}5 & 34 & \text { I } 8\end{array}$ & to $30 . \times 9$ & $-2 \quad 19.7$ & 20.2 & $\mathbf{M}$ & 5.60 & 9.657 & $+r_{5} 425 \mathrm{I} . \mathrm{I}$ & 0.744 & $+1.5^{2}-15.0$ & 8 \\
\hline
\end{tabular}

Luglio 24. Coda debolissima; nucleo $6^{\mathrm{m}}$. Coda S $60^{\circ} \mathrm{W}$. - Sett. 15 . Coda sempre debole, nucleo $3^{\mathrm{m}}$. Sett. 2o. Coda sempre debole $\left(\mathrm{N} 20^{\circ} \mathrm{W}\right)$; nucleo $3^{\mathrm{m}}=2^{n}$. - Ott. 2. Nucleo forse $>3^{\mathrm{m}}$. - Ott. 7 . Nucleo forse $>3^{\mathrm{m}}$; coda sempre debole, ma lunga. - Ott. I 3 . Nucleo $3^{\mathrm{m}}$; coda ad occhio nudo di circa $4^{\circ}$.

L'astro ha una luminosità intrinseca grandissima; la sua modesta apparenza ad occhio nudo dipese dalle forti distanze dal sole e dalla terra. Se si assume come attendibile per le comete la consueta legge di variazione dello splendore colle distanze, il che per le comete in molti casi non si verifica, l'astro potrebbe essere osservato, con cannocchiali aventi un apertura di $40 \mathrm{~cm}$, nell'emisfero australe, ancor nell'autunno del $19 \mathrm{r} 8$ in una grandezza del nucleo di circa $13^{\mathrm{ma}}$.

Cometa I9 14a (Kritzinger). Seguito e fine (vedi A. N. 4728 ).

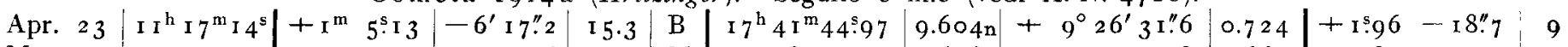

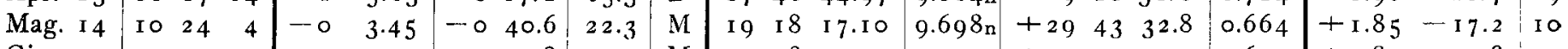

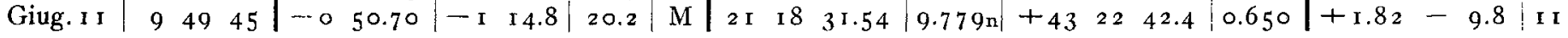
Aprile 23. Nucleo r 2 m. - Maggio I 4. Osservazioni difficili, manca un nucleo ben definito. -- Giugno 1 I. Testa $=0.8, \mathrm{r}^{\mathrm{m}}{ }^{\mathrm{m}} \mathrm{2}, \quad \mathrm{B}=$ E. Bianchi.

Stelle di confronto.

\begin{tabular}{|c|c|c|c|c|c|c|c|}
\hline \multirow{2}{*}{\multicolumn{3}{|c|}{$\begin{array}{c}\alpha \text { I914.0 } \\
5^{\mathrm{h}} 37^{\mathrm{m}} 43^{\mathrm{s}} \cdot 3 \mathrm{o}\end{array}$}} & & 1914 & & \multirow{3}{*}{\multicolumn{2}{|c|}{\begin{tabular}{l}
\multicolumn{1}{c}{ Autorità } \\
Lu 2894 \\
Cbr M. 3565
\end{tabular}}} \\
\hline & & & +36 & 21 & I 5.9 & & \\
\hline IO & I & 21.94 & +49 & 45 & $53 \cdot 7$ & & \\
\hline 10 & 39 & 46.80 & +48 & 59 & $49 . \mathrm{I}$ & Bo 7742 & orr.) \\
\hline I I & 32 & 37.16 & +46 & 37 & 10.0 & Bo 8120 & $\gg$ \\
\hline I 2 & 7 & 9.05 & +44 & 2 & $2 \mathrm{I} .2$ & Bo $83^{87}$ & 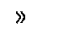 \\
\hline
\end{tabular}

Roma, I9I 4 Novembre I8.

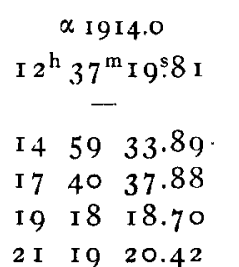

2 I I $\quad 20.42$
8 1914.0 $+4 I^{\circ} \quad 3^{\prime} \quad 5^{\prime \prime} 4$

$+{ }_{15} 4525.8$

$+9337.5$

$+294430.6$

$+43247.0$
Autorità Bo 8621 (korr.) $\mathrm{BD}+36^{\circ} 2353$

Berl A. $543^{8}$

Lpz II 8022

Cbr E. 9866

Bo I 5406 (korr.)

E. Millosevich.

\section{Ephemeride des Kometen 1914 a (Kritzinger).}

\begin{tabular}{|c|c|c|c|c|c|c|c|}
\hline \multirow{2}{*}{$\begin{array}{l}1915 \\
\text { Febr. }\end{array}$} & $\alpha$ vera & 8 vera & $\log \Delta$ & 1915 & $\alpha$ vera & $\delta$ vera & $\log د$ \\
\hline & $a^{\mathrm{h}} 25^{\mathrm{m}} 16^{\mathrm{s}}$ & $+2^{\circ} 28^{\prime} 3$ & 0.605 & Febr. I 6 & $o^{\mathrm{h}} 38^{\mathrm{m}} 3 \mathrm{o}^{\mathrm{s}}$ & $+3^{\circ} 21{ }^{\prime} 5$ & 0.632 \\
\hline 6 & $27 \quad 28$ & 37.0 & & I 8 & $40 \quad 42$ & 30.6 & \\
\hline 8 & 2941 & 45.7 & 0.614 & 20 & $42 \quad 54$ & 39.7 & 0.640 \\
\hline 10 & 3153 & 254.5 & & 22 & 456 & 48.9 & \\
\hline 12 & 346 & $3 \quad 3.5$ & 0.623 & 24 & $\circ 47$ I 8 & $+35^{8 . I}$ & 0.648 \\
\hline 14 & $\begin{array}{lll}0 & 36 & 18\end{array}$ & +312.4 & & & Größe etv & $\mathrm{I} 7^{\mathrm{m}}$ & \\
\hline
\end{tabular}

Kopenhagen, Univ.-Sternwarte, I 9 I 5 Febr. 3.

N. C. Olesen.

Inhalt zu Nr. 4782. R. Klumak. Die Spezialbewegungen der auf Parallaxe untersuchten Sterne. 89. - E. Millosevich. Osservazioni di comete. 103. - N. C. Olesen. Ephemeride des Kometen 1914 a (Kritzinger). 103. 